\title{
Letter to the Editor: Relationship Between Neuropsychiatric Symptoms and Activities of Daily Living in Alzheimer Disease
}

We have read the original article by Ryu et al.," "Relationship between Neuropsychiatric Symptoms and Activities of Daily Living in Alzheimer's Disease", with great interest. Ryu and colleagues reviewed the medical records of 60 patients with suspected Alzheimer disease (AD) and analyzed the relationships between the Neuropsychiatric Inventory (NPI) and 2 functional measures - Instrumental Activities of Daily Living and the Barthel Index. The total NPI score was significantly associated with both functional measures and was a significant predictor of functional status in $A D$, beyond the contribution of cognitive impairment alone. Specifically, neuropsychiatric symptoms were associated with increased functional impairments in AD.

$A D$ patients may have noncognitive dysfunctions, including personality and behavioral changes, delusions, hallucinations, and sleep disorders, as well as cognitive dysfunctions, including impairment in memory, language ability, time and space perception, and judgment. ${ }^{2}$ In 1997, the International Psychogeriatric Association identified these noncognitive dysfunctions as "behavioral and psychological symptoms of dementia" (BPSD). ${ }^{3)}$ The occurrence of BPSD is a significant concern for subjects with moderate to severe dementia, ${ }^{4)}$ as BPSD are associated with increased functional impairment in $A D$. Additionally, BPSD can be assessed with the use of the NPI.

We recruited 103 patients (34 men and 69 women) with AD from the Wonkwang University Ansan Municipal Geriatric Hospital. Patients who underwent neuropsychological tests

\footnotetext{
San Hak $\mathrm{Yi}^{1}$, Seung-Han $\mathrm{Suk}^{2}$

${ }^{1}$ Department of Neurology, Wonkwang University School of Medicine, Institute of Wonkwang Medical Science and Regional Cardiocerebrovascular Center, Iksan, 'Department of Neurology, Wonkwang University Sanbon Medical Center and Wonkwang University Ansan Municipal Geriatric Hospital, Gunpo, Korea
}

Corresponding Author: Seung-Han Suk, MD

Department of Neurology, Wonkwang University Sanbon Medical Center and Wonkwang University Ansan Municipal Geriatric Hospital, 327 Sanbon-ro, Gunpo 15865, Korea

Tel: +82-63-859-1410, Fax: +82-63-842-7379

E-mail: suksh@wku.ac.kr

Received: February 27, 2018

Revised: March 13, 2018

Accepted: March 13, 2018
(Korean version of Mini-Mental State Examination [K-MMSE], Clinical Dementia Rating [CDR], and Bayer-Activities of Daily Living Scale $[\mathrm{B}-\mathrm{ADL}])$ and who met the Diagnostic and Statistical Manual of Mental Disorders, 4th Edition, Text Revision diagnostic criteria were included in this study. We investigated whether antipsychotics were administered and what types of antipsychotics were administered for each patient. These findings were also stratified by dementia severity. There were no significant age- or sex-related differences associated with the use of antipsychotic drugs. However, the K-MMSE, CDR, and $\mathrm{B}-\mathrm{ADL}$ scores were significantly lower in the group taking antipsychotic drugs (Table 1). In addition, in the evaluation of the relationship between dementia severity as assessed by the CDR score and frequency of antipsychotic use, the frequency of antipsychotic use was found to significantly increase with an increase in the CDR score (Table 1). We understand that BPSD are associated with cognitive function and thus agree with Ryu et al. ${ }^{1)}$

The occurrence of BPSD is a major challenge for family members who provide care for patients with dementia. In addition, behavioral and psychological problems lead to increased emotional and physical burden of familial and formal caregivers and result in decreased quality of life for patients

Table 1. Effects of the use versus nonuse of antipsychotics on neuropsychological test results

Nonuse $(n=78) \quad$ Use $(n=25) \quad p$-value

Neuropsychological tests

$\begin{array}{lrrr}\text { K-MMSE } & 16.5 \pm 6.2 & 13.3 \pm 5.7 & <0.05 \\ \text { CDR } & 1.7 \pm 1.2 & 2.6 \pm 1.2 & <0.01 \\ \text { B-ADL } & 16.3 \pm 4.6 & 13.2 \pm 4.6 & <0.05\end{array}$

By K-MMSE score

$\begin{array}{llr}\geq 20(\mathrm{n}=35) & 31(39.7) & 4(16) \\ 10-19(\mathrm{n}=47) & 32(41.0) & 15(60) \\ \leq 9(\mathrm{n}=21) & 15(19.3) & 6(24)\end{array}$

By CDR score

$\begin{array}{llr}\leq 1(\mathrm{n}=43) & 39(50) & 4(16) \\ 2(\mathrm{n}=30) & 21(26.9) & 9(36) \\ 3(\mathrm{n}=30) & 18(23.1) & 12(48)\end{array}$

Values are presented as mean \pm standard deviation or number (\%). K-MMSE, Korean version of Mini-Mental State Examination; CDR, Clinical Dementia Rating; B-ADL, Bayer-Activities of Daily Living Scale.

Copyright (C) 2018 by The Korean Geriatric Society

This is an open access article distributed under the terms of the Creative Commons Attribution Non-Commercial License (http://creativecommons.org/licenses/by-nc/4.0/) which permits unrestricted non-commercial use, distribution, and reproduction in any medium, provided the original work is properly cited. 
and their families. Therefore, pharmacological and nonpharmacological management of BPSD would be helpful in improving functional status and reducing the familial burden of providing care for patients with dementia.

Conflicts of Interest Disclosures: The researchers claim no conflicts of interest.

\section{REFERENCES}

1. Ryu SY, Lee SB, Kim TW, Song IU, Oh EY, Choi HY. Relationship between neuropsychiatric symptoms and activities of daily living in Alzheimer's Disease. J Korean Geriatr Soc 2010;14:131-8.

2. Mega MS, Cummings JL, Fiorello T, Gornbein J. The spectrum of behavioral changes in Alzheimer's disease. Neurology 1996;46: 130-5.

3. Finkel SI, Costa e Silva J, Cohen G, Miller S, Sartorius N. Behavioral and psychological signs and symptoms of dementia: a consensus statement on current knowledge and implications for research and treatment. Int Psychogeriatr 1996;8 Suppl 3:497500 .

4. Bianchetti A, Ranieri P, Margiotta A, Trabucchi M. Pharmacological treatment of Alzheimer's Disease. Aging Clin Exp Res 2006; 18:158-62. 